\title{
Family Support Groups for family members of mentally ill
}

\section{offenders: Therapeutic factors}

\begin{abstract}
This study investigated therapeutic factors of a Family Support Group (FSG) intervention for family members of mentally ill offenders. 17 family members completed the 'Group Therapeutic Factors-Client Questionnaire' (GTF-CQ-28) during 4 sessions of 2 FSGs. Results indicated that families have experienced the relational climate, interactional confirmation, expressing and experiencing mutual positive feelings, forgetting own problems, hope from seeing progress in others, guidance from therapists, and getting interpersonal feedback as helpful over the course of treatment. The therapeutic factors learning by observation, support from the group, and universality of problems correlated with a decrease in self-blame, improved emotional well-being, and experiencing less loss of control over one's life, respectively. Further, family members were satisfied with what the FSG has provided for them personally and for the relation with their relative. Finally, clinical implications of the FSG are discussed. The study sheds light on valuable therapeutic factors within an FSG and the important role of the therapists.
\end{abstract}

\section{Keywords}

Family Support Group, Family Therapy, Family members, Mentally ill offenders, Forensic psychiatry, Therapeutic process, Quantitative research 


\section{INTRODUCTION}

Family members of mentally ill offenders experience a range of emotional, financial and psychological burdens and a double stigma, because their relative is not only seen as a psychiatric patient but also as a criminal (Maclnnes \& Watson, 2002; [reference blinded for review]; Tsang, Pearson, \& Yuen, 2002). This is further aggravated by the fact that, until recently, mentally ill offenders - even when they only committed a minor offense - have often been incarcerated in correctional settings (e.g., prison), because of a lack of sufficient treatment facilities in (forensic) psychiatric settings (Abracen, Gallo, Looman, \& Goodwill, 2015; Sheehy et al., 2016; Vandevelde et al., 2011; Aga, Vander Laenen, Vandevelde, Vermeersch, \& Vanderplasschen, 2017). As a result, the family members often become even more socially isolated, with little or no support from family, friends or professionals (Nordström, Kullgren, \& Dahlgren, 2006; Ridley et al., 2014).

Despite their need for social or professional help (Nordström, Kullgren, \& Dahlgren, 2006; Ridley et al., 2014), there is still a dearth of family interventions to support family members of mentally ill offenders (Absalom, McGovern, Gooding, \& Tarrier, 2010; Absalom-Hornby, Gooding, \& Tarrier, 2011; Geelan \& Nickford, 1999). Nevertheless, family work, and especially Multi-Family Group (MFG) therapy, within and across cultures (e.g., Marlborough multi-family groups, FiSch, Wilows family group ...) and for different psychiatric disorders (e.g. psychotic disorders, mood disorders, and eatings disorders) is growing (Gelin, Cook-Darzens, \& Hendrick, 2017; Potter \& Mannings, 2012). To address this lack, a Family Support Group (FSG) intervention has recently been developed to support and empower family members in dealing with the mental illness and the judicial situation of a relative ([reference blinded for review]). Preliminary results of a pilot study investigating family burdens and cognitive coping strategies 
indicated that family members experienced less self-blame, improved emotional well-being, and less loss of control over their lives after participating in the FSG ([reference blinded for review]). However, it is also important to investigate the therapeutic factors underlying these benefits, which is the major aim of the current study, so that they can be emphasized in therapy.

Research concerning therapeutic factors of family interventions, especially MFG therapy, is lacking in forensic psychiatric research. However, several therapeutic factors of MFG therapies have been identified in various psychiatric and somatic, non-forensic patient groups (Gelin et al., 2017). These factors include: the exchange of experiences through peer support, therapeutic alliance, group cohesion and support, empathy, feeling understood, learning by observation and identification with other families, experiencing communality, gaining insight and hope, and self-disclosure (Gelin et al., 2017; Hellemans et al., 2011; Lemmens, Eisler, Dierick, Lietaer, \& Demyttenaere, 2009; Lemmens et al., 2003). Moreover, the occurrence of these therapeutic factors tends to increase over the course of the intervention (Lemmens et al., 2009). Finally, MFG therapy - which, by its unique structure and non-stigmatizing atmosphere, facilitates communication and social interactions (McFarlane, 2002) - is generally well accepted by its participants. Most studies show an explicitly high treatment satisfaction, in different populations, which is also reflected in low drop-out figures (Brunaux, \& Cook-Darzens, 2008; Salaminiou, Campbell, Simic, Kuipers, \& Eisler, 2015).

The present study specifically focuses on the therapeutic factors of a Family Support Group for relatives of mentally ill offenders. The study has 3 primary aims:

- First, to identify helpful therapeutic factors in an FSG. 
- Second, to investigate their evolution over time and to investigate which therapeutic factors are associated with previously reported treatment improvements (e.g., less self-blame, improved emotional well-being and less loss of control over their lives)

- Finally, to assess the participants' treatment satisfaction.

\section{METHODOLOGY}

\section{Sample selection}

To recruit participants for the FSGs in this study, an information leaflet was disseminated in different settings. These settings were assumed to provide contact with family members of mentally ill offenders in Flanders, such as (forensic) psychiatric facilities, prisons, non-profit organizations for family members of persons with a mental illness, and outpatient mental health services. Family members of mentally ill offenders were selected to participate based on the following inclusion criteria: (1) having a relative with a current or past internment measure ${ }^{i}$ who has a diagnosis of schizophrenia, psychotic or bipolar disorder, and (2) being 18 or older. The inclusion criteria were based on the rationale that MFG therapy is an effective treatment for families living with patients with bipolar and psychotic disorders (Asen, 2002; McFarlane, 2002). Further, we have focused on this patient population because these patients, until a recent change of the law, were often placed under an internment measure after conducting minor 'public' offenses, particularly when they had a long psychiatric history of non-treatment compliance (Aga et al., 2017; Vandevelde et al., 2011). The current living and treatment conditions of the mentally ill relative, the familial background, and the nature of the criminal offense played no role in the selection of the family members. Although the needs of families may differ between families depending on the current living condition of the mentally ill relative (e.g. prison versus forensic psychiatric clinic) or the type of index offense 
(e.g. theft versus murder or intrafamilial violence), no information was available about the nature of the criminal offence or whether the family members were also victimized by their mentally ill relative. An FSG cycle was initiated once a minimum number of 6 family members agreed to participate.

The Ethics Committee of the Ghent University Hospital approved the study (EC decision: B670201526897); and participants gave informed consent before the start of the FSG.

\section{Participants}

In total, 20 family members (mean age of 59.5 years; SD: 12.66 , range: $28-75$ years) were selected and participated in two different FSGs. However, only 19 started the treatment, as one father dropped out before the start of the first session, (due to health issues). During treatment, a second person dropped out after session 1 and a third person dropped out after session 2 (both were mothers). These latter two persons cited 'personal reasons' without further explaining why they were stopping their participation in the FSG. The results of the remaining 17 family members were analysed since they participated in more than one session. Twelve of them were female, with most of them being a mother of a mentally ill offender ( $n$ $=9)$. Others were sisters $(n=2)$ and a daughter. Four fathers and one brother participated. Within the first group cycle, 10 persons from 9 different families participated. The second group cycle consisted of 7 persons from 6 different families. In both groups, one couple participated. On average, family members participated in 3.2 out of 4 sessions (SD: 1.6, range 1-4). 


\section{Organization of the Family Support Groups}

The FSG, based on a systemic MFG therapy format, has been adjusted to the forensic context (Lemmens, Eisler, Migerode, Heireman, \& Demyttenaere, 2007; [reference blinded for review]). In contrast with the original model, our FSG mainly focussed on supporting the family members in dealing with their own difficulties in their relationship with the mentally ill relative (e.g., violent behaviour, burden of care, ...) and their problems with the judicial procedures (e.g., police, court proceedings, internment measure, ...), and not on symptom improvement or rehabilitation of the patient. The group was led by both an MFG therapist (as in the original format) and by an expert in forensic psychiatry, combining (multi)family and forensic expertise ([reference blinded for review]). A group cycle consisted of 4 sessions, with 3 sessions held every two weeks and one session after one month. The content of the sessions focused on some prescribed themes: self-care (session 1), caring in the future (session 2), and strengths of family members (session 3). Session 4 reflected on the strengths discussed in session 3 and contained questions to the family members about the themes of the previous sessions. Homework assignments were given to the participants after each session. For example, a homework assignment about self-care was given after the first session: the therapists asked the family members to do something that they would not ordinarily do in the next two weeks, indulging themselves with something that would help them take a respite from care for a moment and that would make them happy. Each session lasted about 120 minutes with a coffee break after 90 minutes. After the break, the therapists summarized the session and explained the homework assignment. For more information on the protocol of the study, see [reference blinded for review]. 


\section{Data collection}

Therapeutic factors

Participants were asked to complete the 'Group Therapeutic Factors - Client Questionnaire' (GTF-CQ-28) after each session. The 'GTF-CQ-28' is a 28-item questionnaire that asks about helpful experiences during the group sessions (Dierick \& Lietaer, 2008; Lemmens et al., 2009). The GTF-CQ-28 is a short version of the Group Therapeutic Factories-Client Questionnaire (GTF-CQ), which was originally developed to detect therapeutic processes in group therapy using different therapeutic models and group formats. The scales of both questionnaires, particularly of the GTF-CQ-28 in MFG therapies, show high to very high internal consistency and homogeneity and good internal validity (Dierick, 2000; Dierick \& Lietaer, 2002; Dierick \& Lietaer, 2008, Lemmens et al., 2009).

Each of the 28 items of the GTF-CQ-28 represents a Basic therapeutic factor ('Bf 1-28') (see Table 1). These therapeutic factors are hierarchically structured into 7 Main factors (Mf): (Mf 1) Group cohesion, (Mf 2) Interactional confirmation, (Mf 3) Self-revelation, (Mf 4) Selfinsight and progress, (Mf 5) Observational experiences, ( $\mathrm{Mf} 6$ ) Getting directives, (Mf 7) Interactional confrontation. These Main factors are combined into 2 dimensions of therapeutic factors:

(1) Relational Climate of the group (RC),

(2) Psychological Work (PW).

More specifically, RC and PW each contain 10 scales (see Note of Table 1) (Dierick \& Lietaer, 2008). Cronbach Alphas in this study for both domains and Main therapeutic factors over all 4 sessions range from .70 to .89 , except for the Main therapeutic factor 'Interactional confrontation' (Mf 7), because no Cronbach Alpha could be calculated as ' $\mathrm{Bf} 25^{\prime}$ ' had the same 
score (namely, ' 1 ') over the course of treatment (Table 1). Therefore, Mf 7 is not used in the analysis and the underlying subscales of this factor - 'getting interpersonal feedback' (Bf 24) and 'expressing negative feelings' (Bf 25) - are classified into separate scales (Table 1). The occurrence of the experiences or events was rated on a 4-point Likert scale: not applicable (1), slightly applicable (2), clearly applicable (3), highly applicable (4). Mean scores of both domains and therapeutic factors were calculated.

\section{Treatment outcome}

Emotional well-being and loss of control over their lives were measured by the scores on the Zarit Burden Interview (ZBI-22) (Zarit, Reever, \& Bach-Peterson, 1980; Zarit, Todd, \& Zarit, 1986; Mapi Research Trust, 2014), which measures perceived family burdens. Self-blame was measured by using the Cognitive Emotion Regulation Questionnaire (CERQ) (Garnefski, \& Kraaij, 2007; Garnefski, Kraaij, \& Spinhoven, 2006), which focuses on cognitive coping strategies. Both questionnaires have a high reliability and internal validity (Garnefski et al., 2006; Seng et al., 2010). The questionnaires were filled out by the family members one week before the start of the group (T0) and one week after the last session (T1). Improvement from T0 to T1 was described as a significant mean difference of the scores on the ZBI-22 and the CERQ (for further details on the outcome of the study, see [reference blinded for review]).

\section{Treatment satisfaction}

After the group intervention, the participants completed a Post-Evaluation Family Support Group Questionnaire (Post-evaluation FSGQ) to measure treatment satisfaction during the course of treatment (from session 1 to session 4). The questions asked to what extent they perceived the treatment to have been helpful personally and for their relationship with their 
family member. Satisfaction was rated on a 6-point Likert scale (' $1=$ very unsatisfied', '2 = unsatisfied' , '3 = slightly unsatisfied', '4 = slightly satisfied', ‘5 = satisfied' , '6 = very satisfied').

\section{Data analysis}

To conduct the analysis, the assumption of normality was tested, to be able to use parametric tests. A Linear Mixed Model (LMM) analysis was chosen because parameters are allowed to vary over individuals, meaning that the analysis deals with missing values (Verbeke, 1997). The analysis was performed to compare the means of the therapeutic factors and the two domains - Relational Climate (RC) and Psychological Work (PW) - over the 4 sessions. The independent variable 'Time' (sessions 1, 2, 3 and 4) was entered in the model as a fixed effect. Intercepts were considered as fixed effects. Post-hoc pairwise comparisons were computed using Bonferonni correction. To assess model adequacy, residual analysis was performed.

To investigate the association between therapeutic factors and treatment improvement on both family burdens (ZBI-22) and cognitive coping strategies (CERQ), two-tailed Pearson Correlation coefficients were computed. Differences between sessions 1 and 4 were calculated and associated with the mean differences pre- and post-intervention on the ZBI-22 and the CERQ. A descriptive analysis was performed based on the results of the Postevaluation Family Support Group Questionnaire.

The above-mentioned statistical analysis was performed using SPSS 23 with $p<.05$ as standard for statistical significance. 
Table 1 Differences in the mean applicability of main and basic therapeutic factors of FSG $1(n=10)$ and FSG $2(n=7)$

\begin{tabular}{|c|c|c|c|c|c|c|}
\hline Group therapeutic factors & $\begin{array}{l}\text { Session } 1 \\
\text { Mean } \\
{[95 \% \mathrm{Cl}]}\end{array}$ & $\begin{array}{c}\text { Session } 2 \\
\text { Mean } \\
{[95 \% \mathrm{Cl}]}\end{array}$ & $\begin{array}{c}\text { Session } 3 \\
\text { Mean } \\
{[95 \% \mathrm{Cl}]}\end{array}$ & $\begin{array}{c}\text { Session } 4 \\
\text { Mean } \\
{[95 \% \mathrm{Cl}]}\end{array}$ & $\begin{array}{c}\text { Session } 1 \text { to } 4 \\
\text { Mean }\end{array}$ & $\boldsymbol{P}$ \\
\hline A. Relational climate $(\mathrm{RC})^{\mathrm{a}}$ & $\begin{array}{c}2.67 \\
{[2.36,2.98]}\end{array}$ & $\begin{array}{c}2.64 \\
{[2.36,2.92]}\end{array}$ & $\begin{array}{c}2.95 \\
{[2.65,3.24]}\end{array}$ & $\begin{array}{c}2.86 \\
{[2.50,3.23]}\end{array}$ & 2.78 & $.032^{c}$ \\
\hline Mf 1 - Group Cohesion & $\begin{array}{c}3.19 \\
{[2.85,3.52]}\end{array}$ & $\begin{array}{c}3.12 \\
{[2.83,3.41]}\end{array}$ & $\begin{array}{c}3.36 \\
{[3.10,3.61]}\end{array}$ & $\begin{array}{c}3.36 \\
{[3.10,3.61]}\end{array}$ & 3.26 & .300 \\
\hline Acceptance (Bf 1) & $\begin{array}{c}3.17 \\
{[2.78,3.56]} \\
\end{array}$ & $\begin{array}{c}3.18 \\
{[2.85,3.50]} \\
\end{array}$ & $\begin{array}{c}3.34 \\
{[2.93,3.75]}\end{array}$ & $\begin{array}{c}3.38 \\
{[3.04,3.73]}\end{array}$ & 3.27 & .791 \\
\hline $\begin{array}{l}\text { Cohesive working group } \\
\text { (Bf 2) }\end{array}$ & $\begin{array}{c}3.34 \\
{[2.97,3.71]}\end{array}$ & $\begin{array}{c}3.18 \\
{[2.85,3.50]}\end{array}$ & $\begin{array}{c}3.65 \\
{[3.37,3.92]}\end{array}$ & $\begin{array}{c}3.42 \\
{[3.10,3.75]}\end{array}$ & 3.40 & .100 \\
\hline $\begin{array}{l}\text { Support from the group } \\
\text { (Bf 3) }\end{array}$ & $\begin{array}{c}3.02 \\
{[2.63,3.41]}\end{array}$ & $\begin{array}{c}3 \\
{[2.59,3.41]}\end{array}$ & $\begin{array}{c}3.08 \\
{[2.68,3.48]}\end{array}$ & $\begin{array}{c}3.17 \\
{[2.87,3.48]}\end{array}$ & 3.07 & .613 \\
\hline $\begin{array}{l}\text { Mf } 2 \text { - Interactional } \\
\text { confirmation }\end{array}$ & $\begin{array}{c}2.04 \\
{[1.70,2.37]}\end{array}$ & $\begin{array}{c}2.08 \\
{[1.77,2.39]}\end{array}$ & $\begin{array}{c}2.84 \\
{[2.48,3.21]}\end{array}$ & $\begin{array}{c}2.68 \\
{[2.24,3.13]}\end{array}$ & 2.41 & $.000^{d}$ \\
\hline $\begin{array}{l}\text { Expressing mutual } \\
\text { positive feelings ( } \mathrm{Bf} 4)\end{array}$ & $\begin{array}{c}1.17 \\
{[0.89,1.45]}\end{array}$ & $\begin{array}{c}1.18 \\
{[0.90,1.45]}\end{array}$ & $\begin{array}{c}3.16 \\
{[2.68,3.64]}\end{array}$ & $\begin{array}{c}2.55 \\
{[1.98,3.12]}\end{array}$ & 2.01 & $.000^{d}$ \\
\hline $\begin{array}{l}\text { Experiencing positive } \\
\text { feelings (Bf 5) }\end{array}$ & $\begin{array}{c}2.51 \\
{[2.02,2.99]}\end{array}$ & $\begin{array}{c}2.65 \\
{[2.20,3.09]}\end{array}$ & $\begin{array}{c}2.78 \\
{[2.29,3.26]}\end{array}$ & $\begin{array}{c}2.93 \\
{[2.53,3.32]}\end{array}$ & 2.72 & $.042^{c}$ \\
\hline $\begin{array}{l}\text { Self-confidence in } \\
\text { helping others }(\mathrm{Bf} 6)\end{array}$ & $\begin{array}{c}2.42 \\
{[1.86,2.97]}\end{array}$ & $\begin{array}{c}2.41 \\
{[1.93,2.89]}\end{array}$ & $\begin{array}{c}2.50 \\
{[2.03,2.97]}\end{array}$ & $\begin{array}{c}2.54 \\
{[1.97,3.11]}\end{array}$ & 2.47 & .844 \\
\hline Mf 3 - Self-revelation & $\begin{array}{c}2.52 \\
{[2.13,2.90]}\end{array}$ & $\begin{array}{c}2.53 \\
{[2.21,2.85]}\end{array}$ & $\begin{array}{c}2.42 \\
{[1.94,2.89]}\end{array}$ & $\begin{array}{c}2.55 \\
{[2.01,3.09]}\end{array}$ & 2.50 & .837 \\
\hline $\begin{array}{l}\text { Authentic self- } \\
\text { expression (Bf 7) }\end{array}$ & $\begin{array}{c}2.70 \\
{[2.28,3.12]}\end{array}$ & $\begin{array}{c}2.71 \\
{[2.31,3.10]}\end{array}$ & $\begin{array}{c}2.67 \\
{[2.13,3.20]}\end{array}$ & $\begin{array}{c}2.70 \\
{[2.11,3.29]}\end{array}$ & 2.69 & .998 \\
\hline Self-disclosure (Bf 8) & $\begin{array}{c}2.51 \\
{[1.97,3.06]}\end{array}$ & $\begin{array}{c}2.47 \\
{[1.99,2.96]}\end{array}$ & $\begin{array}{c}2.12 \\
{[1.57,2.67]}\end{array}$ & $\begin{array}{c}2.61 \\
{[2.06,3.17]}\end{array}$ & 2.43 & .334 \\
\hline
\end{tabular}




\begin{tabular}{|c|c|c|c|c|c|c|}
\hline $\begin{array}{l}\text { Getting things off one's } \\
\text { chest (Bf 9) }\end{array}$ & $\begin{array}{c}2.36 \\
{[1.94,2.78]}\end{array}$ & $\begin{array}{c}2.41 \\
{[1.93,2.89]}\end{array}$ & $\begin{array}{c}2.48 \\
{[1.89,3.06]}\end{array}$ & $\begin{array}{c}2.53 \\
{[1.95,3.12]}\end{array}$ & 2.44 & .959 \\
\hline \multicolumn{7}{|l|}{ SEPARATE SCALES } \\
\hline $\begin{array}{l}\text { Confidence in therapists } \\
\text { (Bf 10) }\end{array}$ & $\begin{array}{c}3.31 \\
{[2.93,3.70]}\end{array}$ & $\begin{array}{c}3.12 \\
{[2.81,3.43]}\end{array}$ & $\begin{array}{c}3.12 \\
{[2.73,3.52]}\end{array}$ & $\begin{array}{c}3.11 \\
{[2.68,3.55]}\end{array}$ & 3.16 & .755 \\
\hline $\begin{array}{l}\text { Understanding others } \\
\text { (Bf 11) }\end{array}$ & $\begin{array}{c}2.56 \\
{[2.12,3]}\end{array}$ & $\begin{array}{c}2.53 \\
{[2.08,2.98]}\end{array}$ & $\begin{array}{c}2.69 \\
{[2.06,3.32]}\end{array}$ & $\begin{array}{c}2.47 \\
{[1.90,3.03]}\end{array}$ & 2.56 & .688 \\
\hline $\begin{array}{l}\text { Forgetting own } \\
\text { problems through } \\
\text { helping others ( } \mathrm{Bf} 12 \text { ) }\end{array}$ & $\begin{array}{c}1.95 \\
{[1.54,2.36]}\end{array}$ & $\begin{array}{c}1.76 \\
{[1.38,2.15]}\end{array}$ & $\begin{array}{c}2.32 \\
{[1.74,2.91]}\end{array}$ & $\begin{array}{c}2.33 \\
{[1.81,2.85]}\end{array}$ & 2.09 & $.031^{c}$ \\
\hline B. Psychological work (PW) ${ }^{\text {b }}$ & $\begin{array}{c}1.85 \\
{[1.61,2.09]}\end{array}$ & $\begin{array}{c}1.87 \\
{[1.64,2.10]}\end{array}$ & $\begin{array}{c}2.10 \\
{[1.84,2.36]}\end{array}$ & $\begin{array}{c}2.10 \\
{[1.75,2.46]}\end{array}$ & 1.98 & .125 \\
\hline $\begin{array}{l}\text { Mf } 4 \text { - Self-insight and } \\
\text { progress }\end{array}$ & $\begin{array}{c}1.67 \\
{[1.40,1.94]}\end{array}$ & $\begin{array}{c}1.78 \\
{[1.53,2.03]}\end{array}$ & $\begin{array}{c}1.77 \\
{[1.55,1.99]}\end{array}$ & $\begin{array}{c}1.92 \\
{[1.56,2.28]}\end{array}$ & 1.78 & .209 \\
\hline $\begin{array}{l}\text { Making progress in } \\
\text { trying out new } \\
\text { behaviour (Bf13) }\end{array}$ & $\begin{array}{c}1.31 \\
{[1.06,1.56]}\end{array}$ & $\begin{array}{c}1.35 \\
{[0.95,1.76]}\end{array}$ & $\begin{array}{c}1.41 \\
{[1.09,1.73]}\end{array}$ & $\begin{array}{c}1.80 \\
{[1.23,2.37]}\end{array}$ & 1.47 & .062 \\
\hline $\begin{array}{l}\text { Insight into patient's } \\
\text { problem (Bf 14) }\end{array}$ & $\begin{array}{c}2.14 \\
{[1.55,2.73]}\end{array}$ & $\begin{array}{c}2 \\
{[1.49,2.51]}\end{array}$ & $\begin{array}{c}2.03 \\
{[1.56,2.50]}\end{array}$ & $\begin{array}{c}2.11 \\
{[1.51,2.72]}\end{array}$ & 2.07 & .829 \\
\hline $\begin{array}{l}\text { Insight into connections } \\
\text { between current } \\
\text { behaviour and feelings } \\
\text { and childhood } \\
\text { experiences (Bf 15) }\end{array}$ & $\begin{array}{c}1.20 \\
{[0.99,1.41]}\end{array}$ & $\begin{array}{c}1.23 \\
{[1.01,1.46]}\end{array}$ & $\begin{array}{c}1.09 \\
{[0.96,1.22]}\end{array}$ & $\begin{array}{c}1.18 \\
{[0.91,1.44]}\end{array}$ & 1.17 & .139 \\
\hline
\end{tabular}




\begin{tabular}{|c|c|c|c|c|c|c|}
\hline $\begin{array}{l}\text { Becoming conscious of } \\
\text { existential responsibility } \\
\text { (Bf 16) }\end{array}$ & $\frac{2}{[1.62,2.38]}$ & $\begin{array}{c}2.53 \\
{[2.12,2.94]}\end{array}$ & $\begin{array}{c}2.47 \\
{[1.97,2.96]}\end{array}$ & $\begin{array}{c}2.54 \\
{[2.04,3.03]}\end{array}$ & 2.38 & .181 \\
\hline $\begin{array}{l}\text { Mf 5-Observational } \\
\text { experiences }\end{array}$ & $\begin{array}{c}2.87 \\
{[2.53,3.21]}\end{array}$ & $\begin{array}{c}2.75 \\
{[2.43,3.06]}\end{array}$ & $\begin{array}{c}2.96 \\
{[2.62,3.30]}\end{array}$ & $\begin{array}{c}3.01 \\
{[2.60,3.43]}\end{array}$ & 2.90 & .093 \\
\hline $\begin{array}{l}\text { Learning by observation } \\
\text { (Bf 17) }\end{array}$ & $\begin{array}{c}2.68 \\
{[2.22,3.13]}\end{array}$ & $\begin{array}{c}2.88 \\
{[2.48,3.28]}\end{array}$ & $\begin{array}{c}2.80 \\
{[2.27,3.34]}\end{array}$ & $\begin{array}{c}2.96 \\
{[2.45,3.46]}\end{array}$ & 2.83 & .701 \\
\hline $\begin{array}{l}\text { Similarity with others (Bf } \\
18)\end{array}$ & $\begin{array}{c}3.24 \\
{[2.77,3.71]}\end{array}$ & $\begin{array}{c}3.18 \\
{[2.76,3.59]}\end{array}$ & $\begin{array}{c}3.13 \\
{[2.69,3.56]}\end{array}$ & $\begin{array}{c}3.18 \\
{[2.67,3.68]}\end{array}$ & 3.18 & .929 \\
\hline $\begin{array}{l}\text { Hope from seeing } \\
\text { progress in others } \\
\text { (Bf 19) }\end{array}$ & $\begin{array}{c}2.15 \\
{[1.72,2.57]}\end{array}$ & $\begin{array}{c}1.94 \\
{[1.56,2.33]}\end{array}$ & $\begin{array}{c}2.58 \\
{[2.01,3.14]}\end{array}$ & $\begin{array}{c}2.67 \\
{[2.16,3.18]}\end{array}$ & 2.33 & $.045^{c}$ \\
\hline $\begin{array}{l}\text { Universality of problems } \\
\text { (Bf 20) }\end{array}$ & $\begin{array}{c}3.40 \\
{[2.98,3.83]}\end{array}$ & $\begin{array}{c}3 \\
{[2.55,3.44]}\end{array}$ & $\begin{array}{c}3.34 \\
{[2.88,3.80]}\end{array}$ & $\begin{array}{c}3.13 \\
{[2.58,3.68]}\end{array}$ & 3.22 & .226 \\
\hline Mf 6 - Getting directives & $\begin{array}{c}1.90 \\
{[1.56,2.25]}\end{array}$ & $\begin{array}{c}2.23 \\
{[1.99,2.48]}\end{array}$ & $\begin{array}{c}2.25 \\
{[1.88,2.62]}\end{array}$ & $\begin{array}{c}2.34 \\
{[1.92,2.76]}\end{array}$ & 2.18 & .050 \\
\hline $\begin{array}{l}\text { Suggestions from } \\
\text { members (Bf 21) }\end{array}$ & $\begin{array}{c}1.64 \\
{[1.26,2.02]}\end{array}$ & $\begin{array}{c}1.71 \\
{[1.46,1.95]}\end{array}$ & $\begin{array}{c}2.07 \\
{[1.51,2.63]}\end{array}$ & $\begin{array}{c}2.12 \\
{[1.66,2.77]}\end{array}$ & 1.88 & .232 \\
\hline $\begin{array}{l}\text { Learning a method to } \\
\text { master a problem (Bf } \\
22 \text { ) }\end{array}$ & $\begin{array}{c}1.64 \\
{[1.22,2.06]}\end{array}$ & $\begin{array}{c}1.94 \\
{[1.56,2.33]}\end{array}$ & $\begin{array}{c}1.68 \\
{[1.24,2.12]}\end{array}$ & $\begin{array}{c}1.81 \\
{[1.34,2.27]}\end{array}$ & 1.77 & .384 \\
\hline $\begin{array}{l}\text { Guidance from therapist } \\
\text { (Bf 23) }\end{array}$ & $\begin{array}{c}2.42 \\
{[1.99,2.85]}\end{array}$ & $\begin{array}{c}3.06 \\
{[2.67,3.44]}\end{array}$ & $\begin{array}{c}3.04 \\
{[2.63,3.44]}\end{array}$ & $\begin{array}{c}3.02 \\
{[2.56,3.47]}\end{array}$ & 2.88 & $.017^{c}$ \\
\hline
\end{tabular}




\begin{tabular}{|c|c|c|c|c|c|c|}
\hline $\begin{array}{l}\text { Getting interpersonal } \\
\text { feedback (Bf 24) }\end{array}$ & $\begin{array}{c}1.45 \\
{[1.12,1.78]}\end{array}$ & $\begin{array}{c}1.47 \\
{[1.15,1.79]}\end{array}$ & $\begin{array}{c}2.50 \\
{[1.91,3.10]}\end{array}$ & $\begin{array}{c}2.26 \\
{[1.77,2.75]}\end{array}$ & 1.92 & $.009^{d}$ \\
\hline $\begin{array}{l}\text { Expressing negative } \\
\text { feelings ( } \mathrm{Bf} 25 \text { ) }\end{array}$ & $\begin{array}{c}1 \\
{[1,1]}\end{array}$ & $\begin{array}{c}1 \\
{[1,1]}\end{array}$ & $\begin{array}{c}1 \\
{[1,1]}\end{array}$ & $\begin{array}{c}1 \\
{[1,1]}\end{array}$ & 1 & - \\
\hline Modelling (Bf 26) & $\begin{array}{c}2.18 \\
{[1.60,2.75]}\end{array}$ & $\begin{array}{c}2.06 \\
{[1.63,2.48]}\end{array}$ & $\begin{array}{c}2.48 \\
{[1.86,3.08]}\end{array}$ & $\begin{array}{c}2.55 \\
{[1.95,3.16]}\end{array}$ & 2.32 & .153 \\
\hline $\begin{array}{l}\text { Recognizing own } \\
\text { transference reactions } \\
\text { (Bf 27) }\end{array}$ & $\begin{array}{c}1.62 \\
{[1.13,2.10]}\end{array}$ & $\begin{array}{c}1.41 \\
{[1.09,1.73]}\end{array}$ & $\begin{array}{c}1.71 \\
{[1.12,2.30]}\end{array}$ & $\begin{array}{c}1.42 \\
{[0.99,1.84]}\end{array}$ & 1.54 & .378 \\
\hline $\begin{array}{l}\text { Open expression of } \\
\text { feelings towards } \\
\text { therapist ( } \mathrm{Bf} 28 \text { ) }\end{array}$ & $\begin{array}{c}1.82 \\
{[1.30,2.33]}\end{array}$ & $\begin{array}{c}1.53 \\
{[1.16,1.90]}\end{array}$ & $\begin{array}{c}1.68 \\
{[1.07,2.28]}\end{array}$ & $\begin{array}{c}1.70 \\
{[1.15,2.25]}\end{array}$ & 1.68 & .507 \\
\hline
\end{tabular}

Note: $M f=$ Main Therapeutic Factor; $B f=$ Basic Therapeutic Factor; $C l=$ Confidence Interval; $P=$ significance based on F-test, Likert scale of the GTF-CQ-28: '1 = not applicable', '2 = slightly applicable', '3 = clearly applicable', '4 = extremely applicable'

${ }^{a}$ Relational Climate consists of the following ten Basic Therapeutic Factors: Bf 1, 2, 3, 4, 5, 6, 7, 8, 10, 11

${ }^{b}$ Psychological Work consists of the following ten Basic Therapeutic Factors: Bf 13, 14, 15, 16, 20, 21, 22, 24, 26, 27

${ }^{c} p<.05$

${ }^{d} p<.01$ 


\section{RESULTS}

\section{Therapeutic factors rated as helpful by the participants over the 4 sessions}

From the first session, a wide variety of therapeutic factors were experienced as helpful (score $\geq 3$ ) by the family members over the 4 group sessions. Most frequently reported therapeutic factors within the group's relational climate $(\mathrm{RC})$ were: group cohesion $(\mathrm{Mf} 1)$, acceptance (Bf 1), cohesive working group (Bf 2), support from the group (Bf 3) and confidence in therapists (Bf 10). Within the domain of Psychological Work (PW), only the basic factors - similarity with others (Bf 18) and universality of problems ( $B f 20)$ - were frequently mentioned as helpful. The domain RC was overall more frequently mentioned as helpful than the domain PW (RC: $M=2.78, S D=0.56$ and $P W: M=1.98, S D=0.47 ; t(16)=10.13, p<0.01)$.

Two therapeutic factors were also rated as not strongly applicable (score $\leq 1.5$ ): insight into connections between current behaviour and feelings and childhood experiences (Bf 15), and recognizing one's own transference reactions ( $B f$ 27). Moreover, all of the participants always rated expressing negative feelings ( $B f 25$ ) as ' 1 ' during the different group sessions - meaning that family members did not express negative feelings during the group interventions (see Table 1).

\section{Changes in the perception of therapeutic factors over time}

The dimension 'Relational Climate' $(F(3,14.731)=3.872, p<.05)$, and the main therapeutic factor 'interactional confirmation' (Mf 2$)(F(3,14.319)=17.601, p<.01)$ within this dimension, were increasingly frequently mentioned as helpful over the course of the 4 sessions (see Table 1). A statistically significant increase was also found for the basic therapeutic factors, consistent increases were found for 'expressing mutual positive feelings' $(B f 4)(F(3,13.586)=$ 
32.961, $p<.01)$, 'experiencing positive feelings' (Bf 5) $(F(3,14.834)=3.505, p<.05)$, 'forgetting own problems through helping others' $(B f 12)(F(3,15.039)=3.868, p<.05)$ within $R C$. Within the dimension PW, only the basic therapeutic factors 'hope from seeing progress in others' $(B f 19)(F(3,14.460)=3.441, p<.05)$, 'guidance from therapists' $(B f 23)(F(3,14.781)=4.717, p$ $<0.5)$ and 'getting interpersonal feedback' (Bf 24) $(F(3,14.886)=5.530, p<.01)$ showed an increase over time (Table 1).

Association between therapeutic factors and treatment improvement on family burdens (ZBI-22) and cognitive coping strategies (CERQ)

Associations were analysed by correlating the mean difference between pre- and postintervention scores with the difference of the main and basic therapeutic factors over the course of the intervention (i.e. mean difference of session 4 - session 1). Based on the scores of the CERQ, a significant decrease in self-blame was found after the intervention (preintervention: $M=8.88$ (SD: 2.87 ) versus post-intervention: $M=7.53$ (SD: 2.53$), F(1,169)=$ $5.493, p<.05$ ), indicating that family members make less use of this coping strategy [reference blinded for review]. This result was correlated with different therapeutic factors over the course of the intervention (session $4-$ session 1 ). Significant correlations were found with 'learning by observation' ( $\mathrm{Bf} 17)(r=0.537, \mathrm{p}<.05)$, 'insight into patient's problem' (Bf 14) $(r$ $=0.529, \mathrm{p}<.05)$ and 'modelling' (Bf 26) $(r=0.645, \mathrm{p}<.01)$.

The scores on the ZBI-22 showed an improvement of emotional well-being after the intervention (pre-intervention: $M=13.53(S D=5.18$ ) versus post-intervention: $M=12.29$ (SD $=4.80), F(1,165)=7.643, p<.05)$ ([reference blinded for review]). This indicates that people experience fewer family burdens, which increases their emotional well-being. Over the time of the intervention (session 4-session 1), a negative correlation with 'support from the group' 
(Bf 3) $(r=-0.590, p<.05)$ was found. A decrease on the scale 'loss of control over one's life' was found after the intervention (pre-intervention: $M=9(S D=2.98)$ versus post-intervention: $M=8.12(S D: 3.04), F(1,163)=4616, p<.05)$ ([reference blinded for review]) and indicates that people experience less family burden on this scale, which increases the sense of control they have over their lives. From session 1 to session 4, negative correlations were found with 'similarity with others' $(\mathrm{Bf} 18)(r=-0.563, \mathrm{p}<.05)$ and with 'universality of problems' $(\mathrm{Bf} 20)(\mathrm{r}$ $=-0.533, p<.05)$.

\section{Treatment satisfaction}

Based on the descriptive analysis of the results of the Post-evaluation FSGQ, family members reported to be satisfied over the course of the FSG with what the intervention provided for them personally (M: 5.56; SD: 0.51). Furthermore, they were also satisfied with what the intervention has provided for the relationship they have with their mentally ill relative (M: 5; SD: 0.63).

\section{DISCUSSION}

We have conducted two FSGs to support and empower family members of mentally ill offenders. After treatment, they reported less self-blame, improved emotional well-being and less loss of control over their lives ([reference blinded for review]). The current study investigated which therapeutic factors are mentioned by the group participants as helpful in an FSG and are associated with beneficial outcome. Our study showed that family members participating in an FSG experience a variety of therapeutic factors as helpful. The most mentioned therapeutic factors were: group cohesion, cohesive working group, acceptance, 
support from the group, confidence in therapists and experiencing similarity with others and universality of problems.

These results are in line with several other studies of MFG therapy in psychiatric patient populations, indicating that group cohesion and support, similarity with others and universality of problems are important therapeutic factors in family groups (Asen, \& Schuff, 2006; Lemmens et al., 2003; Lemmens et al., 2009; McFarlane, 2002). The latter therapeutic processes may help family members feel less stigmatized and more supported, when coming into contact with peers confronted with similar problems (Asen \& Schuff, 2006). In our study, some therapeutic factors such as 'expressing negative feelings' were less frequently reported. This may partly reflect the use of the FSG format, which focused on people's capabilities and strengths rather than on negative feelings (Lemmens et al., 2009). The FSG format may have created less room for family members to openly discuss negative feelings or experiences.

Furthermore, most applicable therapeutic factors - such as 'group cohesion', 'acceptance', 'cohesive working group', 'support from the group', 'similarity with others' and 'universality of problems' - are continuously reported as very important right from the start (session 1) and in all successive sessions. This may partly reflect the needs of the participating population of family members in our study. Indeed, family members of mentally ill offenders often feel strongly isolated, chronically burdened and stigmatised by the mental illness and criminal offence, and lacked professional and social support both in our groups as well as in international research studies (Nordström, Kullgren, \& Dahlgren, 2006; [reference blinded for review]; Tsang, Pearson, \& Yuen, 2002). Yet, other therapeutic factors - such as 'interactional confirmation', 'expressing mutual positive feelings', 'experiencing positive feelings', 'forgetting own problems through helping others', 'hope from seeing progress in others', 'guidance from 
therapists' and 'getting interpersonal feedback' - gradually become more frequently reported over the course of the intervention. In contrast with the "continuously reported" therapeutic factors, these evolving therapeutic factors are mostly situated within the interactional domain, as most reported factors are about helping others and expressing positive feelings, getting feedback and making progress in seeing and trying out new behaviour. This might reflect the fact that interactional patterns, which are often stimulated in an MFG therapy (Gelin, Cook-Darzens, \& Hendrick, 2017), need some time to develop in an FSG. This may also partly be explained by the protocol that was used - with a different treatment focus for each session - and the absence of the mentally ill offender in the treatment sessions ([reference blinded for review]).

In our study the decrease of the participants' self-blame correlated with the fact that they could learn new behaviour (e.g., 'learning by observation' and 'modelling') or gain new insights ('insight into patient's problem') within the FSG. These results are partly in line with the study of Lemmens and colleagues (2009) showing improvements in depressive symptoms when the patients experienced different kinds of behavioural interventions or activation such as trying out new behaviour, learning by observation and modelling in MFG therapy. Indeed, MFG therapy offers multiple opportunities for family members to indirectly learn from each other, to gain new insights and to experiment with new behaviours (Eisler, 2005; McFarlane, 2002). It may help them to develop new behavioural and cognitive coping strategies, such as selfreflection or acceptance, for which they are able to rebuild self-confidence (Lemmens et al., 2003). Although other studies also mention getting support as a factor helpful in reducing feelings of self-blame, this was not observed in our study (Moses, 2010; Nordström et al., 2006; Ridley et al., 2014). Perhaps due to the small number of sessions, a correlation between 
self-blame and experiencing support did not have time to develop. But, this may also be explained by differences in participation population (e.g., families within a forensic psychiatric mental health context or the predominant presence of mothers in the group).

Interestingly, therapeutic factors such as 'group support', 'similarity with others' and 'universality of problems' - which are most frequently mentioned in MFG therapy literature (Lemmens et al., 2003; Lemmens et al., 2009) - are associated with less family burden in this study. The presence of other family members in the group generates a supportive climate, enabling family members to recognize similarities and differences in stories (Hellemans et al., 2011). Experiencing communality by feeling supported and similar to others makes family members feel less isolated and stigmatised within this study but also in international research (Asen, \& Schuff, 2006; Lemmens et al., 2003; McFarlane, 2002).

The findings of this study may further indicate that different therapeutic factors are associated with different outcomes in MFG therapy. Both therapists and family members can experience different therapeutic factors as helpful in a family discussion group, because a variety of learning levels is available: specifically, individual, family and group levels (Depestele, Claes, Dierickx, Colman, Schoevaerts, \& Lemmens, 2017; Lemmens et al., 2003). Furthermore, starting from session 2 , therapeutic guidance is rated as clearly applicable by the participants.

The primary results of an FSG show that family members were satisfied, which is also reflected in the low dropout rates (Salaminiou et al., 2015). Furthermore, an FSG can be seen as effective, because therapeutic factors considering interactional processes evolve through treatment and families are able to gain insight into their situation. Family members can come 
into contact with others in similar situations, which heightens feelings of support and solidarity. As many family members feel socially isolated and doubly stigmatised, an FSG can help to rebuild social networks (McFarlane, 2002). Although we have seen that some therapeutic factors become more helpful through treatment, 4 sessions may be too short and for this reason, long-term FSGs should be developed and studied in the future. Further, it would be interesting to explore what the implications would be on our results if the family members were allowed to determine the agenda of the sessions ('What might be useful for them to address in these four sessions?'). This may also be a reason why the group members spoke less about the offense that was committed and more about the experienced burden by the psychiatric disorder of their relative. Furthermore, the important role therapists play should not be neglected, and more attention should be given to a strengths-based framework within FSGs.

The results of this study should be interpreted in the context of some limitations, which restrict the generalizability of the results. Important limitations are the small size and the heterogeneity of the sample. The participation in the FSG may be biased as most family members (11 out of 17) were recruited from a non-profit organisation for family members of mentally ill persons. Moreover, participants needed to contact the researcher, which may have been perceived as a 'big step' by family members who have often become mistrustful in contacting professionals. Most of the participating family members already had a long history of taking care of the mentally ill offender and have learned how to cope with it. In the study, no control condition was initiated, so it cannot be decisively concluded that the changes in therapeutic factors are due to an effect of the intervention and/or other variables. Because of the small sample, the effect of the number of sessions on the reported results was not 
examined, but in future research this could be important to investigate with larger samples. No data was available for the mentally ill relative. Finally, no information was obtained about to what extent the family members' needs were addressed in treatment.

Despite the limitations, this study sheds light on helpful therapeutic factors as reported by family members of mentally ill offenders when attending an FSG. Future (randomized and controlled) studies should further elaborate on FSGs and therapeutic processes, with attention to family members' needs and therapist perceptions.

\section{Acknowledgements}

[will be added after the review process] 


\section{REFERENCES}

[references blinded for review will be added after the review process]

Absalom, V., McGovern, J., Gooding, P. A., \& Tarrier, N. (2010). An assessment of patient need for family intervention in forensic services and staff skill in implementing family interventions. The Journal of Forensic Psychiatry and Psychology, 21(3), 350-365. doi: $10.1080 / 14789940903426893$

Abracen, J., Gallo, A., Looman, J., \& Goodwill, A. (2015). Individual Community-Based Treatment of Offenders With Mental Illness: Relationship to Recidivism. Journal of Interpersonal Violence, 31(10), 1842-1858. doi: 10.1177/088620515570745

Absalom-Hornby, V., Gooding, P., \& Tarrier, N. (2011). Coping with schizophrenia in forensic services: the needs of relatives. Journal of Nervous and Mental Disease, 199(6), 398-402. doi: 10.1097/NMD.0b013e31821cd394

Aga, N., Vander Laenen, F., Vandevelde, S., Vermeersch, E., \& Vanderplasschen, W. (2017). Recovery of Offenders Formerly Labeled as Not Criminally Responsible: Uncovering the Ambiguity From First-Person Narratives. International Journal of Offender Therapy and Comparative Criminology. Doi: 10.1177/0306624X17730617

Asen, E. (2002). Multiple family therapy: an overview. Journal of Family Therapy, 24(1), 3-16. doi: $10.1111 / 1467-6427.00197$

Asen, E., \& Schuff, H. (2006). Psychosis and multiple family group therapy. Journal of Family Therapy, 28(1), 58-72. doi: 10.1111/j.1467-6427.2006.00337.x

Bishop, P., Clilverd, A., Cooklin, A., \& Hunt, U. (2002). Mental health matters: a multi-family framework for mental health intervention. Journal of Family Therapy, 24(1), 31-45. doi: $10.1111 / 1467-6427.00199$ 
Brunaux, F., \& Cook-Darzens, S. (2008). La thérapie multifamiliale: une alternative à la thérapie unifamiliale dans le traitement de l'anorexie mentale de l'enfant et de l'adolescent? Thérapie familiale, 29(1), 87-102. doi: 10.3917/tf.081.0087

Depestele, L., Claes, L., Dierckx, E., Colman, R., Schoevaerts, K., \& Lemmens, G. (2017). An Adjunctive Multi-Family Group Intervention with or without Patient Participation during an Inpatient Treatment for Adolescents with an Eating Disorder: A Pilot Study, European Eating Disorders Review, 25(6), 570-578. doi: 10.1002/erv.2556

Dierick, P. (2000). Cliëntperceptie van therapeutische factoren in groepspsychotherapie en groeigroepen: een structureel en vergelijkend onderzoek [Client perception of therapeutic factors in group psychotherapy and growth groups: A structural and comparative research]. Non-published doctoral dissertation, Katholieke Universiteit Leuven.

Dierick, P., \& Lietaer, G. (2002). De Groeps Therapeutische Factoren-Cliënt Vragenlijst (GTFCV): psychometrische en klinische karakteristieken van het structuurmodel [The Group Therapeutic Factories-Client Questionnaire (GTF-CQ): Psychometric and clinical characteristics of the structural model]. In T.J.C. Berk et al. (Eds.), Handboek groepspsychotherapie, (Q6: 1-70). Houten/Zaventem: Bohn Stafleu Van Loghum.

Dierick, P., \& Lietaer, G. (2008). Client Perception of Therapeutic Factors in Group Psychotherapy and Growth Groups: An Empirically-Based Hierarchical Model. International Journal of Group Psychotherapy, 58(2), 203-230. doi: 10.1521/ijgp.2008.58.2.203

Eisler, I. (2005). The empirical and theoretical base of family therapy and multiple family day therapy for adolescent anorexia nervosa. Journal of Family Therapy, 27(2), 104-131. doi: 10.1111/j.1467-6427.2005.00303.x 
Garnefski, N., \& Kraaij, V. (2007). The Cognitive Emotion Regulation Questionnaire Psychometric Features and Prospective Relationships with Depression and Anxiety in Adults. European Journal of Pscyhological Assessment, 23(3), 141-149. doi: $10.1027 / 1015-5759.23 .3 .141$

Garnefski, N., Kraaij, V., \& Spinhoven, P. (2006, version 1.03). Manual for the use of the Cognitive Emotion Regulation Questionnaire (Dutch version). Leiderdorp, The Netherlands: DATEC.

Geelan, S., \& Nickford, C. (1999). A survey of the use of family therapy in medium secure units in England and Wales. The Journal of Forensic Psychiatry, 10(2), 317-324. doi: $10.1080 / 09585189908403685$

Gelin, Z, Cook-Darzens, S., \& Hendrick, S. (2017). The evidence base for Multiple Family Therapy in psychiatric disorders: a review (part 1). Journal of Family Therapy, [Online Version of Record published before inclusion in an issue]. doi: 10.1111/14676427.12178

Heimans, H., Vander Beken, T., Schipaanboord, A.E. (2015). Eindelijk een echte nieuwe en goede wet op de internering? Deel 1: De gerechtelijke fase. Rechtskundig Weekblad, 79(27), 1043-1064

Hellemans, S., De Mol, J., Buysse, A., Eisler, I., Demyttenaere, K., \& Lemmens, G. M. D. (2011). Therapeutic processes in multi-family groups for major depression: Result of an interpretative phenomenological study. Journal of Affective Disorders, 134(1-3), 226234. doi: 10.1016/j.jad.2011.05.050

Lemmens, G. M., Wouters, S., Heireman, M., Eisler, I., Lietaer, G., \& Sabbe, B. (2003). Beneficial factors in family discussion groups of a psychiatric day clinic: perceptions by the 
therapeutic team and the families of the therapeutic process. Journal of Family Therapy, 25(1), 41-63. doi: 10.1111/1467-6427.00234

Lemmens, G. M. D., Eisler, I., Migerode, L., Heireman, M., \& Demyttenaere, K. (2007). Family discussion group therapy for major depression: a brief systemic multi-family group intervention for hospitalized patients and their family members. The Association for Family Therapy and Systemic Practice, 29(1), 49-68. doi: 10.1111/j.14676427.2007.00369.x

Lemmens, G., Eisler, I., Dierick, P., Lietaer, G., \& Demyttenaere, K. (2009). Therapeutic factors in a systemic multi-family group treatment for major depression: patients' and partners' perspectives. Journal of Family Therapy, 31(3), 250-269. doi: 10.1111/j.1467$6427.2009 .00465 . x$

Mapi Research Trust (2014). Zarit Burden Interview version 1.0 - Scaling and scoring version 3.0. Lyon: Mapi Research Trust.

Maclnnes, D. L., \& Watson, J. P. (2002). The differences in perceived burdens between forensic and non-forensic caregivers of individuals suffering from schizophrenia. Journal of Mental Health, 11(4), 375-388. doi: 10.1080/09638230020023741

McFarlane, W. R. (2002). Multifamily Groups in the treatment of severe psychiatric disorders. New York/London: The Guilford Press.

Moses, T. (2010). Exploring Parents' Self-Blame in Relation to Adolescents' Mental Disorders. Family Relations, 59(2), 103-120. doi: 10.1111/j.1741-3729.2010.00589.x

Nordström, A., Kullgren, G., Dahlgren, L. (2006). Schizophrenia and violent crime: the experience of parents. International Journal of Law and Psychiatry, 29, 57-67. doi: 10.1016/j.ijlp.2004.07.002 
Potter, S. \& Mannings, C. (Eds.) (2012). Multi-family therapy: Challenging behaviour in educational settings. Context, 123, pp. 1-57.

Ridley, J., McKeown, M., Machin, K., Rosengard, A., Little, S., Briggs, S., Jones, F., \& Deypurkaystha, M. (2014). Exploring family carer involvement in forensic mental health services. Scotland: Support in Mind Scotland, University of Central Lancashire, \& Forensic Mental Health Services Managed Care Network, 1-87 (paper).

Salaminiou, E., Campbell, M., Simic, M., Kuipers, E., \& Eisler, I. (2015). Intensive multi-family therapy for adolescents anorexia nervosa: an open study of 30 families. Journal of Family Therapy, doi: 10.111/1467-6427.12075

Seng, B. K., Luo, N., Ng, W. Y., Lim, J., Chionh, H. L., Goh, J., \& Yap, P. (2010). Validity and reliability of the Zarit Burden Interview in assessing caregiving burden. ANNALS Academy of Medicine Singapore, 39(10), 758-763.

Sheehy, K., Rehberger, T., O’Shea, A., Hammond, W., Blais, C., Smith, M., Preston White, K. Jr., \& Goodloe, N. (2016). Evidence-Based Analysis of Mentally III Individuals in the Criminal Justice System. IEEE Systems and Information Engineering Design Symposium (SIEDS). Charlottesville, VA: IEEE (pp. 250-254).

Tsang, H. W., Pearson, V., Yuen, C. H. (2002). Family needs and burdens of mentally ill offenders. International Journal of Rehabilitation Research, 25(1), 25-32.

Vandevelde, S., Soyez, V., Vander Beken, T., De Smet, S., Boers, A., \& Broekaert, E. (2011). Mentally ill offenders in prison: The Belgian case. International Journal of Law and Psychiatry, 34(1), 71-78. doi: 10.1016/j.ijlp.2010.11.011

Verbeke G. (1997). Linear Mixed Models for Longitudinal Data. In: Linear Mixed Models in Practice. Lecture Notes in Statistics, vol. 126 (pp. 63-153). New York, NY: Springer. 
Zarit, S. H., Reever, K. E., \& Bach-Peterson, J. (1980). Relatives of the impaired elderly, correlates of feelings of burden. Gerontologist, 20(6), 649-655.

Zarit, S., Todd, P. A., Zarit, \& J. M. (1986). Subjective burden of husbands and wives as caregivers: a longitudinal study. Gerontologist, 26(3), 260-266.

\footnotetext{
' In Belgium, mentally ill offenders can be placed under an 'internment measure'. This is defined as: 'a safety measure to protect society and that simultaneously aims to ensure that the mentally ill offender is provided with the care his/her condition requires in view of his/her reintegration into society' (Heimans, Vander Beken, \& Schipaanboord, 2015, p. 1051, translation by the authors).
} 